\title{
Anisotropic Wave Propagation and Apparent Conductivity Estimation in a Fast Electrophysiological Model: Application to XMR Interventional Imaging
}

\author{
P.P. Chinchapatnam ${ }^{1}$, K. S. Rhode ${ }^{2}$, A. King ${ }^{2}$, G. Gao ${ }^{1}$, \\ Y. $\mathrm{Ma}^{2}$, T. Schaeffter ${ }^{2}$, D. Hawkes ${ }^{1}$, R.S. Razavi ${ }^{2}$, \\ D.L.G. Hill ${ }^{1}$, S. Arridge ${ }^{1}$, and M. Sermesant ${ }^{2,3}$ \\ ${ }^{1}$ University College London, Centre for Medical Image Computing, UK \\ ${ }^{2}$ King's College London, Division of Imaging Sciences, UK \\ ${ }^{3}$ INRIA Sophia Antipolis, Asclepios Team, France
}

\begin{abstract}
Cardiac arrhythmias are increasingly being treated using ablation procedures. Development of fast electrophysiological models and estimation of parameters related to conduction pathologies can aid in the investigation of better treatment strategies during Radio-frequency ablations. We present a fast electrophysiological model incorporating anisotropy of the cardiac tissue. A global-local estimation procedure is also outlined to estimate a hidden parameter (apparent electrical conductivity) present in the model. The proposed model is tested on synthetic and real data derived using XMR imaging. We demonstrate a qualitative match between the estimated conductivity parameter and possible pathology locations. This approach opens up possibilities to directly integrate modelling in the intervention room.
\end{abstract}

\section{Introduction}

Radio-frequency ablation (RFA) techniques are becoming increasingly preferred as an alternative to drug therapy for treatment of cardiac arrhythmias. These procedures are carried out under x-ray guidance, with specialised catheters for making invasive recordings of the electrical activity of the heart. Although RFA procedures can be highly effective with minimal side effects, they still have unsatisfactory success rates for some group of patients. There is still a need for substantial innovation in guiding these interventions. XMR suites are a new type of clinical facility combining in the same room a MR scanner and a mobile cardiac x-ray set. Registration of the two image spaces (MR and x-ray) makes it possible to combine patient anatomy with electrophysiology recordings 1 .

The use of electrophysiology models simulating electrical propagation for various cardiac arrhythmias is a way forward in guiding the RFA procedures. Existing models however are computationally expensive and are presently not suitable for direct use in the intervention room. The aim of this research is to design

${ }^{1}$ Obtained from Ensite (St. Jude Medical) or Carto (Biosense).

N. Ayache, S. Ourselin, A. Maeder (Eds.): MICCAI 2007, Part I, LNCS 4791, pp. 575-583, 2007.

(C) Springer-Verlag Berlin Heidelberg 2007 
electrophysiological models that are suited for clinical use and to propose methods to combine these models with interventional data in order to better estimate the patient cardiac function and aid in the guidance of RFA procedures.

Modelling the complete electrophysiology of the heart begins with the incorporation of electrical phenomena from the microscopic cellular level into the macroscopic set of partial differential equations (PDE) modelling a continuum. The resulting models are the bidomain and monodomain models [1. The numerical solution of these models is computationally demanding due to a very small spatial scale associated with the electrical propagation in comparison to the size of the ventricles. Fortunately as the depolarisation occurs only in a narrow region, the depolarisation region can be considered as a propagating wavefront [2] and an Eikonal equation can be derived describing this activation phenomenon. Further, the solution of these models cannot be directly correlated with pathologies due to the complex interaction of various parameters present in the models. We believe that development of algorithms for identifying the hidden parameters in the electrophysiological models would help cardiologists in a quicker diagnosis and treatment of pathologies. For our interventional purpose and as parameter adjustment often requires several simulations, we propose to use the Eikonal equation to model the electrophysiology.

In this paper, we develop a Fast Marching Method (FMM) for the numerical solution of the anisotropic Eikonal-Curvature (EC) equation on surface triangulations and use it in an iterative algorithm to estimate the apparent conductivity parameter. This parameter is estimated first on a global basis and then local corrections are made. The developed model is validated on synthetic data and also applied to clinical data. We show that the proposed estimation procedure can potentially aid in the detection of scarred/infarcted regions in the myocardium using electrophysiological (Ensite) and geometrical (XMR) information.

\section{Anisotropic Fast Marching Electrophysiology Model}

Cardiac tissue is strongly anisotropic with wave speeds that differ substantially depending on their direction. For example, in human myocardium, propagation is about $0.5 \mathrm{~m} / \mathrm{s}$ along fibres and about $0.17 \mathrm{~m} / \mathrm{s}$ transverse to the fibres. In this section, we present a fast electrophysiological model for depolarisation wave propagation on anisotropic cardiac surfaces.

The static EC equation [3] for the depolarisation time $(T)$ in the myocardium is given by

$$
c \sqrt{k D}\left\{\sqrt{\nabla T^{t} \mathbf{M} \nabla T}\right\}-D\left[\left\{\sqrt{\nabla T^{t} \mathbf{M} \nabla T}\right\} \nabla \cdot\left(\frac{\mathbf{M} \nabla T}{\sqrt{\nabla T^{t} \mathbf{M} \nabla T}}\right)\right]=1,
$$

where $c$ and $k$ are constants related to the cell membrane. $D$ is the volumetric electrical conductivity of the tissue and $\mathbf{M} 2$ is a tensor quantity incorporating

${ }^{2} \mathbf{M}=\mathbf{A} \overline{\mathbf{D}} \mathbf{A}^{t}$, where $\mathbf{A}$ is the matrix defining the fibre directions in the global coordinate system and $\overline{\mathbf{D}}=\operatorname{diag}\left(1, \lambda^{2}, \lambda^{2}\right) . \lambda$ is the anisotropic ratio of propagation speeds and is of the order 0.33 in human myocardium. 
cardiac fibre directions. The term in square brackets in Eq. (1) is the anisotropic curvature flow term.

Sophisticated numerical techniques have already been developed to solve the Eikonal-Curvature equation to study propagation in normal myocardium [234]. These models generate accurate depolarisation times at the expense of high computational cost. However, the requirement for our electrophysiological model is that it should be fast. In accordance to this view, we propose an alternative fixed point iterative method combined with a very fast Eikonal solver based on a modified anisotropic Fast Marching Method (FMM) 5. The fixed point iterative algorithm is presented in Algorithm 1, The curvature flow term in Step 2 is obtained by the anisotropic generalisation of the curvature term given in [6] and the Eikonal equations in Steps 1 and 3 are solved using the modified FMM.

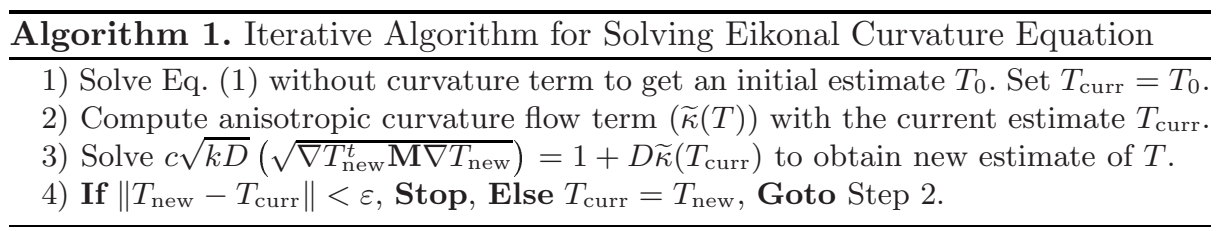

\subsection{Validation of Algorithm}

The algorithm presented to solve Eq. 1 is tested for its convergence and accuracy by performing numerical experiments. A square domain $[0,1] \times[0,1]$ is considered with the initialisation point being the origin. The parameter values used are $c=3.89$ and $D=0.005$. We tested the algorithm on a family of unstructured meshes ranging from $N=121$ to $N=5041$ mesh points. The myocardial tissue is simulated by requiring that the speed of propagation in the $x$-direction (along fibre direction) be three times the speed of propagation in the $y$-direction.

The results of numerical experiments conducted are presented in Fig. 11. The left sub figure (1) A) shows the convergence of the fixed point iterative algorithm for different mesh sizes. From this figure, it can be seen that the fixed point

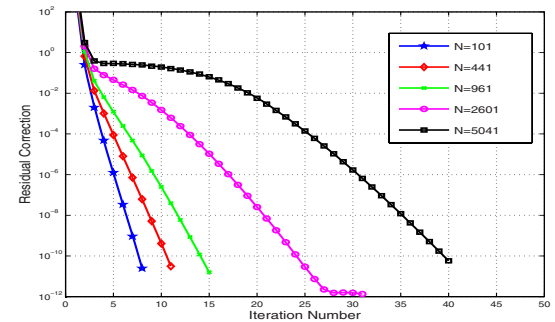

A

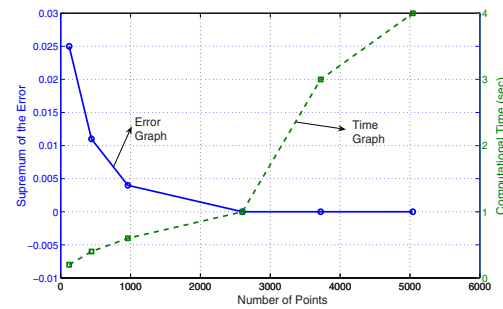

B

Fig. 1. A. Convergence of the fixed point algorithm for different meshes $\left(\left\|T_{k+1}-T_{k}\right\|_{\infty}\right.$ vs $k$ ) B. Evolution of error (thick line) and Wall time (dashed) w.r.t mesh size. 
algorithm converges to a pre-specified limit (say $10^{-10}$ ) for different meshes and also that the convergence seems to be dependent on the number of mesh points $N$. For the densest mesh considered in the study (5000 nodes), the algorithm seems to converge in about 40 iterations which is very fast and hence may be suitable for faster computations in real time interventional cases.

Fig. 1.B shows the convergence of the solution to a fixed limit as $N \rightarrow \infty$ and also the wall time clocked by the FMM algorithm. As the exact solution of this problem is not available, we construct a reference solution by solving the problem on a fine mesh $(N=5041)$ and the convergence of the solution on coarser meshes is examined towards this limit. From the figure, it can clearly be seen that mesh convergence is achieved for this particular case. Note that in comparison to time taken by the sophisticated FEM method (about 4 minutes for 2300 nodes) 2], the FMM method obtains results on a 5000 node mesh in about 4 sec. Further, the stability of the FEM method depends on several terms (diffusion, boundary conditions etc.,) and is strongly dependent on the mesh size thus increasing the computational effort required. In contrast, we observe that the FMM method is quite stable even on coarser meshes and one can refine meshes with a correspondingly small increase in computational effort $(\mathcal{O}(n \log (n)))$ and a reasonable first order accuracy 7 .

\section{Apparent Conductivity Estimation Procedure}

Cardiologists generally base their analysis of electrophysiological data on the isochrones of depolarisation/repolarisation times of the epicardium and endocardium. However, these time variables are difficult to interpret due to the influence of the geometry and curvature of the propagating front and often need expert intervention. We hope that estimation of additional parameter maps related to conduction pathologies 3 would enable cardiologists to perform a quicker diagnosis. Additionally, a model based method has the advantage of allowing the use of the model in a predictive way, once adjusted to the data. This can be very useful to test therapies and plan interventions.

The idea in this section is to estimate these hidden parameters using the proposed anisotropic fast marching EC model and clinical measurements obtained during electrophysiology study. The two parameters that are present in the EC formulation are $c$ (related to cell membrane) and the apparent conductivity $D$. As we have only one measure which is the depolarisation time, we can only chose to estimate one parameter. We aim to estimate the apparent conductivity $D$, which can be thought of as a good indicator in the case of pathologies. For example, in case of a scarred tissue, we expect that the apparent conductivity in the scarred region may be different (lower) than in the normal tissue.

The algorithm is based on matching the isochrones of measured depolarisation time $T^{m}$ (from clinical data) to the simulated depolarisation time $T^{s}$ (from model), in order to get an estimate of the conductivity value $(D)$. To begin

\footnotetext{
${ }^{3}$ A similar goal of estimating myocardial conductivity from body surface potential
} measurements using a nonlinear system approach can be found in [8]. 
with, we assume that there is a nominal value of conductivity ( $D_{\text {global }}$ ) on the entire cardiac surface and then you have local variations in conductivity $\left(D_{\text {local }}\right)$ at each mesh point. We expect that these variations have a large magnitude at points near possible pathological locations.

The global conductivity is estimated by using a simple bisection method (Algorithm 2). As the FMM used in the estimation algorithm is very fast, the global estimation of apparent conductivity parameter is done in a very quick time. The obtained $D_{\text {global }}$ after convergence is used as an initial guess for the local conductivity estimation algorithm.

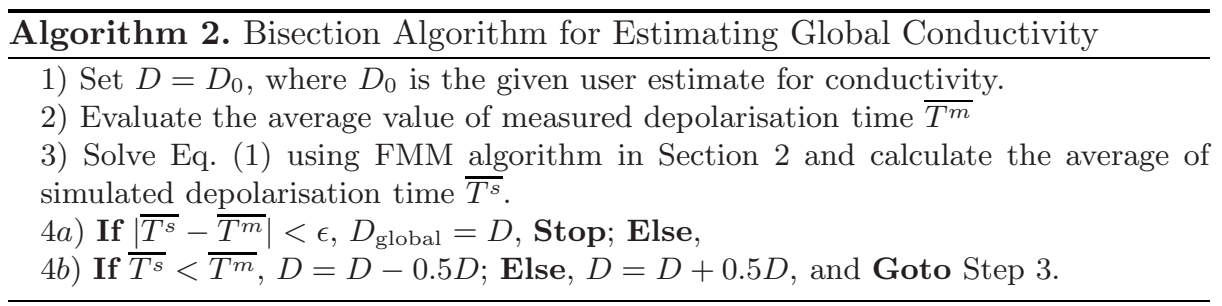

The local correction of conductivity at each mesh point is obtained by minimisation of the quadratic error between the model and data. The algorithm works on minimising the cost function given by $J(\mathbf{D})=\frac{1}{2} \sum_{i=1}^{N}\left(T_{i}(\mathbf{D})-T_{i}^{m}\right)^{2}$, where $T_{i}^{m}$ is the measured depolarisation time at vertex $i$ and $T_{i}(\mathbf{D})$ is the solution of the Eikonal-Curvature equation with the given set of parameters $\mathbf{D}$. The minimisation is done using the method of steepest descent. The gradient vector is obtained using forward finite differences. i.e., $\left(\partial_{D} J\right)_{i}=\left[J\left(\mathbf{D}+\delta \tau_{i}\right)-J(\mathbf{D})\right] / \delta$, where $\tau_{i}$ is the unit vector in the $i^{\text {th }}$ dimension. We note that the algorithm (local estimation) can be expensive for a large number of points. However, this idea of gradient minimisation can be incorporated into a framework in which the conductivity is estimated on region by region basis, where the entire surface is divided into different regions. Further, we expect improved optimisation performance using more powerful descent algorithms such as conjugate gradients.

\subsection{Propagation in a Slab of Tissue}

We evaluate the performance of presented algorithms initially using synthetic data. A two dimensional slab of anisotropic cardiac tissue $([0,1] \times[0,1])$ is considered for computations. The bottom surface represents the endocardium and the top represents the epicardium. The fibre directions are varied according to $\theta(y)=2 \pi y / 3 L$ where $L=1.0$ represents the thickness of myocardium. The initial point of excitation is taken as the bottom leftmost corner of the slab. To simulate the myocardium propagation velocity of around $0.5 \mathrm{~m} / \mathrm{s}$, the value of the parameters taken was $c=3.89$ and $D=0.005$ along the fibre direction 3 and the anisotropy ratio is set to 0.33 . The anisotropic Eikonal-Curvature equation was solved for a case where the conductivity distribution map is taken as shown in Fig. 2. A. Two regions were defined with a higher $\left(2 D_{0}\right)$ and lower 


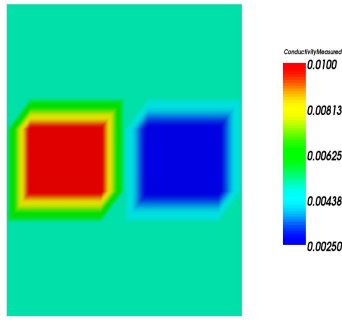

A. Conductivity Map

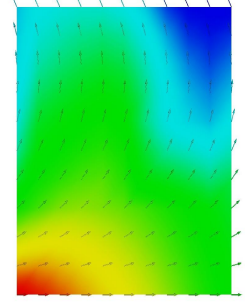

B. Isochrones

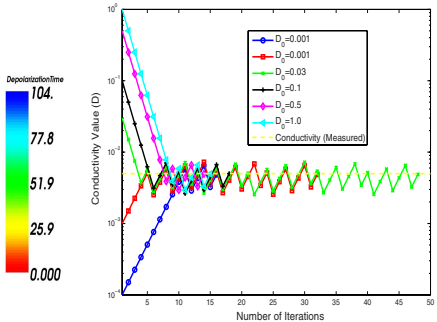

C. $D_{\text {global }}$ Convergence

Fig. 2. A. Simulation of different conductivity zones with $D$ twice the normal value (left square in red) and half the normal value (right square in blue); B. Resulting isochrones with the anisotropic Eikonal-Curvature equation (used as measured data for conductivity estimation); The arrows in the figure represent the fibre direction; C. Convergence of the global conductivity estimation procedure for different user values

$\left(0.5 D_{0}\right)$ conductivities than the nominal conductivity $\left(D_{0}=0.005\right)$ over the entire domain. The resulting isochrones of depolarisation time obtained by using the fast electrophysiological model (shown in Fig.2.B) are taken as the measured data for the conductivity estimation algorithm. Fig. 2. C shows the convergence of the global estimation procedure for different initial user values. From the figure, it can clearly be seen that the nominal conductivity values obtained by the bisection procedure are very near to the nominal conductivity value $\left(D_{0}\right)$ used for obtaining measured data. Thus, the need for a good initial guess by the user is eliminated by this global conductivity estimation algorithm.

Next, we evaluate the performance of the estimation algorithm (global+local) for a case when the fibre directions are not known (Fig. 3. A) and when the fibre directions are given (Fig. 3,B). When the fibre directions are not known, we assume isotropic wave propagation. In the case of isotropic propagation, it can be seen that although the values of the estimated conductivities are not same as the measured values, a fair idea of the location of low conductivity region is obtained. The estimated low conductivity region is more diffused as compared to that in Fig. 2.A. A much sharper delineation of conductivity regions is obtained when the estimation algorithm is run with fibre data (Fig. 3,B). After convergence of the estimation procedure for the anisotropic case, we obtain a mean error of $2.8 \times 10^{-2}$ on the depolarisation times which are between 0 and $104 \mathrm{~ms}$. Further, we tested the estimation algorithm for the anisotropic case with noisy fibre data given by adding Gaussian noise to the fibre directions and the estimated conductivity map is shown in Fig. 3.C4. The estimated conductivity map with noisy fibre data corresponds more closely to the measured conductivity map (Fig. 2. A) than assuming isotropic wave propagation (Fig. 3. A). From these results,

\footnotetext{
${ }^{4}$ A zero mean Gaussian noise with a standard deviation of $10^{\circ}$ was added to the fibre directions. We further note that the percentage change of the estimated conductivity w.r.t the added noise ranging from $0^{\circ}-15^{\circ}$ standard deviations was less than $0.1 \%$. This suggests that the algorithm is quite robust to Gaussian noise.
} 


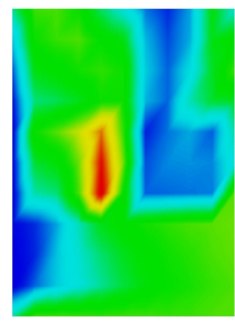

A. Isotropic

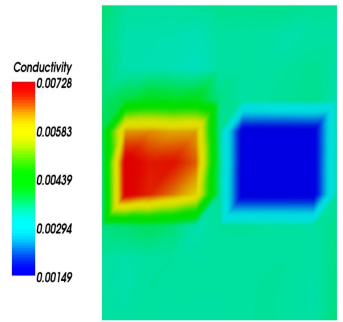

B. Anisotropic

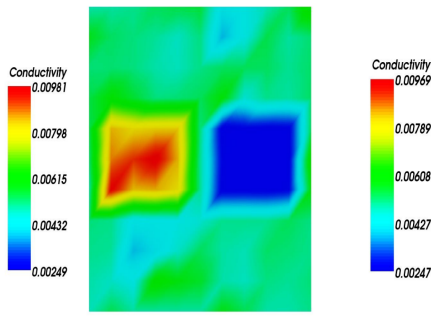

C. Anisotropic (noisy fibre data)

Fig. 3. A. Estimated $D$ with isotropic $\operatorname{FMM}(\lambda=1.0)$; B. Estimated $D$ with anisotropic $\operatorname{FMM}(\lambda=0.33, \theta(y)=2 \pi y / 3 L)$; C. Estimated $D$ with noisy fibre direction

it can be clearly seen that the global and local parameter estimation algorithm can successfully estimate different conductivity regions present in the domain. Further, in relation to clinical applications, as patient specific data on fibre orientations is difficult to obtain in-vivo, prior knowledge on these orientations has to be used. Statistical studies on these showed a variability of around 10 degrees [9, thus we tested the impact of such unknown (fibre orientations) on the estimation procedure.

\section{Application to Clinical Data}

In this section, we present some preliminary results of applying the conductivity estimation algorithm to clinical data obtained during an electrophysiology study in XMR environment. XMR registration makes it possible to incorporate the electrophysiology measurements and patient anatomy in the same coordinate space. This in turn enables to validate the conductivity estimation model developed by comparing the pathology location obtained from MR with estimated low conductivity regions.

The electrical measurements were obtained using the Ensite system, which is a non-contact invasive catheter based device for recording the electrical activity of the heart (reconstructed on 256 points). This data is from a patient with left bundle branch block, so the initialisation comes from the septum rather than the Purkinje network. The fibre orientation on the endocardium is taken as $+60^{\circ}$ to the circumferential axis $(\widehat{\xi})$ 5. We assume that the 3D aspect of electrical propagation does not affect the endocardial surface recordings. The apparent conductivity is estimated on this case and is compared to scar locations estimated by segmentation of late enhancement MR image (see Fig. (4). We obtain a mean error of $6 \times 10^{-2}$ on the depolarisation times which are between 0 and $66 \mathrm{~ms}$.

\footnotetext{
${ }^{5}$ The circumferential direction at each mesh point is obtained by a cross product of the long axis vector $(\widehat{l})$ of the reconstructed geometry and the position vector of the considered point. Unit vectors in the radial $(\widehat{\eta}=\widehat{l} \times \widehat{\xi})$ and longitudinal $(\widehat{\zeta}=\widehat{\xi} \times \widehat{\eta})$ are obtained successively.
} 


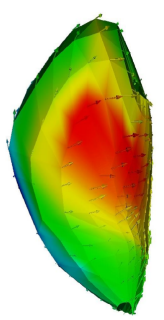

A. Isochrones
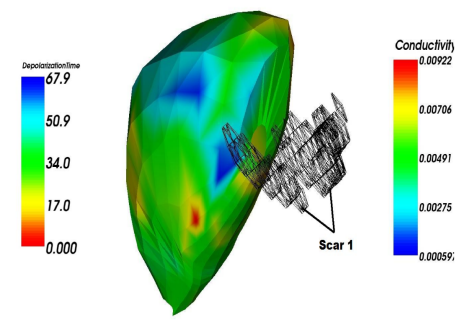

B. Scar 1 Comparison

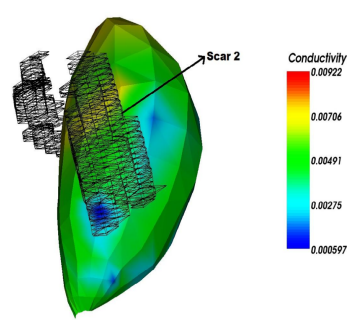

C. Scar 2 Comparison

Fig. 4. A. Isochrones obtained using the anisotropic FMM algorithm; B, C. Matching between the estimated conductivity and scars segmented in a late enhancement MR image. Note that the scars do not lie on the ventricular surface as the late enhancement image was acquired one day before the procedure.

Visual inspection of Fig. 4 4 shows that the low conductivity areas and scars were co-localised to the accuracy of the MRI to Ensite registration.

\section{Conclusion and Future Work}

A novel anisotropic fast marching algorithm incorporating fibre data is presented to achieve fast simulations of electrophysiology, along with a parameter estimation procedure to adjust model parameters using interventional data. The apparent conductivity parameter present in the model is estimated on a global and local basis, to identify possible pathological locations (lower conductivity). The algorithm is used on both synthetic as well as real interventional data and the results obtained are very encouraging. Having such a model opens up possibilities for early detection of possible scar regions in the myocardium and also aid in treatment and planning of different strategies before the actual RFA procedure.

There can be several improvements made to the proposed model to enhance its estimation properties which will be the focus of our future research. In order to model re-entrant arrhythymias, we need to introduce the fast marching in a time-stepping fashion to enable nodes to become repolarised. Initial work demonstrating re-entrant wave behaviour using FMM applied to the classical Eikonal equation was presented in [10. Further in this paper, the cardiac propagation is considered only on triangulated surfaces as relevant clinical data is present only on the endocardial surface. In order to build a more realistic model one should consider the propagation in the entire myocardial volume. A volumetric FMM has been developed for tetrahedral meshes and is currently under evaluation.

\section{References}

1. Sundness, J., Lines, G.T., Cai, X., Nielsen, B.F., Mardal, K.A., Tveito, A.: Computing the Electrical Activity in the Heart. Springer, Heidelberg (2006)

2. Tomlinson, K., Hunter, P., Pullan, A.: A FEM for an eikonal equation model of the myocardial excitation wavefront propagation. SIAM J. Appl. Math. (2002) 
3. Keener, J.P.: An eikonal-curvature equation for action potential propagation in myocardium. J. Math. Biol. 29, 629-651 (1991)

4. Franzone, P.C., Guerri, L., Rovida, S.: Wavefront propagation in activation model of the anisotropic cardiac tissue. J. Math. Biol. (1990)

5. Konukoglu, E., Sermesant, M., Peyrat, J.M., Clatz, O., Delingette, H., Ayache, N.: A recursive anisotropic fast marching approach to reaction diffusion equation: Application to tumor growth modelling. In: Karssemeijer, N., Lelieveldt, B. (eds.) IPMI 2007. LNCS, vol. 4584, Springer, Heidelberg (2007)

6. Barth, T., Sethian, J.: Numerical schemes for the Hamilton-Jacobi and Level Set equations on triangulated domains. J. Comput. Phys. 145, 1-40 (1998)

7. Sethian, J.: Level Set Methods and Fast Marching Methods. CUP (1999)

8. Wang, L.W., Zhang, H.Y., Shi, P.C.: Simultaneous recovery of three-dimensional myocardial conductivity and electrophysiological dynamics: a nonlinear system approach. Computers in Cardiology 33, 45-48 (2006)

9. Peyrat, J.M., Sermesant, M., Delingette, H., Pennec, X., Xu, C., McVeigh, E., Ayache, N.: Towards a statistical atlas of cardiac fiber structure. In: Larsen, R., Nielsen, M., Sporring, J. (eds.) MICCAI 2006. LNCS, vol. 4190, pp. 297-304. Springer, Heidelberg (2006)

10. Sermesant, M., Konukoglu, E., Delingette, H., Coudiere, Y., Chinchapatnam, P., Rhode, K.S., Razavi, R., Ayache, N.: An anisotropic multi-front fast marching method for real-time simulation of cardiac electrophysiology. In: FIMH 2007 (2007) 